\title{
Practical Aspects of Surface Generated Third Harmonic for Highly Precise Beam-workpiece Alignment
}

\author{
Kristian CVECEK $^{* 1,2}$, Johannes STRAUSS ${ }^{2,4}$, Ilya ALEXEEV ${ }^{2,4}$, Isamu MIYAMOTO ${ }^{2,3}$ and Michael SCHMIDT ST, $^{1,2}$ \\ ${ }^{* 1}$ blz-Bayerisches Laserzentrum, Konrad-Zuse Str. 2-6, 91052, Erlangen, Germany \\ E-mail:k.cvecek@blz.org \\ ${ }^{2}$ SAOT - Erlangen Graduate School in Advanced Optical Technologies, Friedrich-Alexander- \\ University of Erlangen-Nuremberg, Paul-Gordan-Str. 6, 91052 Erlangen, Germany \\ ${ }^{3}$ Osaka University, Japan, Osaka University, 2-1 Yamada-Oka, Osaka 565-0871, Japan \\ ${ }^{4}$ Institute of Photonic Technologies, Friedrich-Alexander-University of Erlangen-Nuremberg, Paul- \\ Gordan-Str. 3, 91052Erlangen, Germany
}

\begin{abstract}
Precise beam-workpiece alignment is highly important in laser processing. Though many methods exist for this task few allow high-precision measurements using the processing laser itself. One such method detects the laser's backreflection from the workpiece's surface using a confocal setup and yields ideally an accuracy of about $10 \%$ of the Rayleigh length of the focused beam. Nevertheless, under certain conditions the accuracy of this method may suffer from a mismatch of beam divergences between laser and confocal detection optics. This becomes important when transparent materials are processed in three dimensions using optics with very high numerical apertures or if expensive materials or samples have to be processed where any misalignment would be costly. A possible solution to this is surface based third harmonic generation that allows for absolute surface detection while having the same accuracy as the confocal method. However, this method requires a complex setup while exhibiting low conversion efficiency. Both reasons make a proper alignment of this measurement scheme difficult. Herein we present results showing a clear conversion efficiency dependence on the reflection coefficient of the generating interface for a number of material combinations. This makes the selection of suitable materials more straightforward and reduces the alignment effort. The highest conversion efficiency was achieved for air/silicon which exhibits the highest reflection coefficient of the investigated materials.

DOI: $10.2961 / \mathrm{jlmn} .2013 .03 .0003$
\end{abstract}

Keywords: Laser materials processing, height measurements, diagnostic applications of nonlinear optics, nonlinear optics at surfaces, ultrafast optics

\section{Introduction}

The necessity for precise focus positioning becomes increasingly pronounced the smaller the feature size of the laser generated structure gets. This is typically the case for applications where pulse durations in femtosecond and picosecond range are used such as glass welding by ultrafast lasers [1] or in laser-assisted direct nanostructuring [2]. Commonly, a confocal method is used for sample-beamalignment, where a camera observes the back reflection of the laser beam from the sample's surfaces, comparable to the working principle of a confocal microscope. Albeit this method is fairly straightforward and also takes possible drifts and changes of the transversal laser beam mode into account, it can nonetheless suffer from a possible misalignment of the camera-mode to the laser-beam-mode. To determine such misalignment, a reference workpiece is usually processed at varying focus positions and the produced structures are evaluated in terms of size and depth with respect to the used focus positions. This approach requires in general a subsequent microscope analysis and at least one sacrificial sample, resulting in a fairly complex effort. As described in [3], the camera-laser-mode alignment can be made easier (and non-damaging to the sample) by exploiting a third harmonic generation (THG) on surfaces [4].
The confocal method as well as the surface based third harmonic generation (SBTHG) are both described by the schematics shown in fig. 1. In case of the confocal method the incoming laser beam is focused by the optical system used for processing except that the laser is set to low power so that no processing of the sample occurs. The beam is then partially backreflected by the workpiece and the reflected light is collimated again by the same optical system as used for processing. Even though the mirror used for deflecting the laser beam towards the processing optics is in most cases a dielectric mirror with high reflection coefficient the weak leakage of the backreflected and recollimated laser beam is sufficient enough to be detected by the confocal camera system with a more than acceptable signal to noise ratio. The confocal camera system consists of a focusing optics and a CMOS or CCD sensor whose pixel act as small pinholes having a diameter that lies in the range of the actual pixel size. In order to measure the position of the surface with respect to the laser beam's focus either the focusing optics or the sample is displaced along the optical axis while monitoring the backreflection by the confocal camera system. The brightest pixel for each axial displacement is used for the evaluation of the surface position since the highest brightness (at the smallest spot size at the confocal sensor) is detected when the focal spot of the 
focused laser beam coincides with the surface of the workpiece. As already stated a high measurement accuracy can be achieved only if the the camera-mode and the laserbeam-mode are the same meaning that they have especially the same beam divergence. Is this not the case only reference measurements can be carried out.

As already mentioned the SBTHG based surface detection method is (whenever applicable) optimally suited for absolute measurements or for the calibration of the confocal backreflection method. Here the laser beam normally used for processing is focused by the processing optics onto the (transparent) sample at low power so that no processing occurs (fig. 1). Due to the change of the refractive index at the surface an electric field gradient will develop which is responsible for the SBTHG. As is known from theory and experiments (compare fig. 2) the intensity of the generated third harmonic wave depends strongly on the intensity of the fundamental exhibiting a cubic relationship. The intensity of a gaussian beam reaches its maximum intensity at its focus. Therefore, the highest SBTHG signal can be expected when the focus of the fundamental wave is placed on the sample's surface. Since the intensity of the fundamental is highest at its focus the highest surface generated third harmonic signal is to be expected when the focus of the fundamental coincides with the sample's surface. Any further optical systems such as a collimating lens or a camera lens to reimage the third harmonic signal onto a camera sensor won't have an influence on the detected surface position [3]. Of course, in order to achieve reliable results it is necessary to filter out the fundamental wave from the third harmonic by suitable filters or dichroic mirrors.

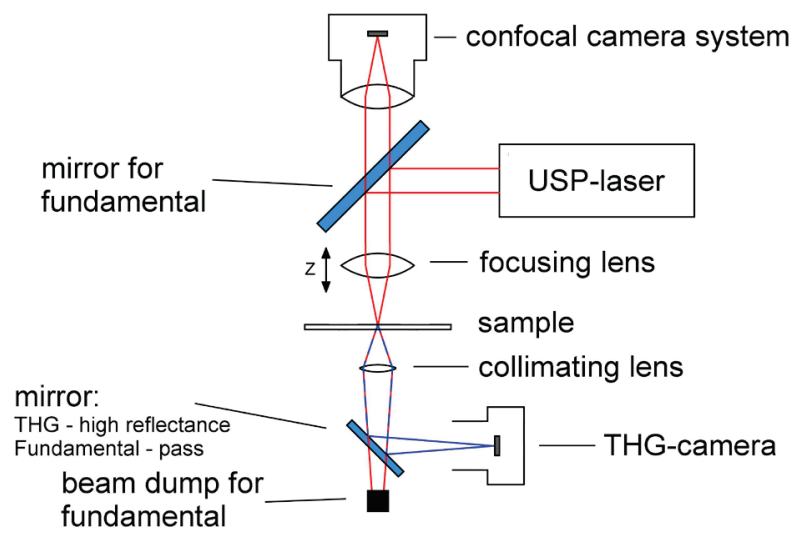

Fig. 1 Schematics of the confocal backreflection and the SBTHG based surface detection setups.

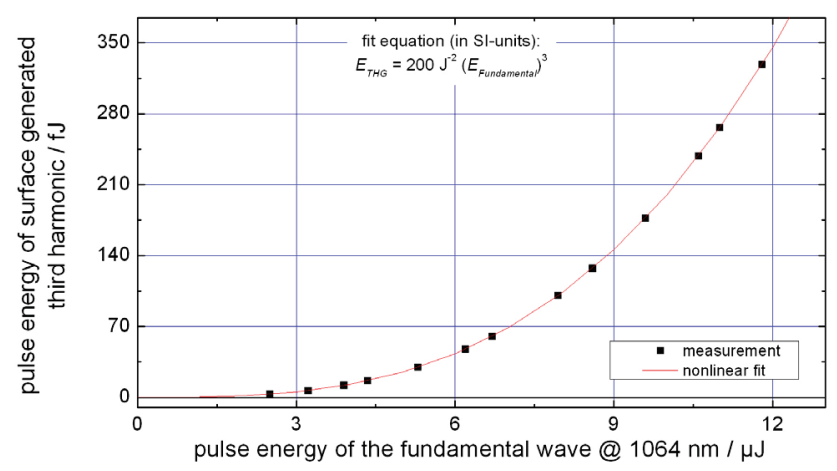

Fig. 2 Conversion efficiency of surface generated third harmonic shown in terms of pulse energy measured on a fused silica sample.
Fig. 2 shows the relationship between the pulse energies of the generated third harmonic wave and the fundamental wave generated at a fused silica / air interface using ultra short pulsed laser at a fundamental wave at a wavelength of $1064 \mathrm{~nm}$ and the pulses having a FWHM-pulse duration of $10 \mathrm{ps}$. The laser beam was focused onto the first surface of the glass sample using a lens with a focal length of $25 \mathrm{~mm}$ resulting in a spot diameter of $20 \mu \mathrm{m}$ on the glass surface. As can be seen in fig. 3 the conversion efficiency is fairly low as only a portion of $3 \times 10^{-8}$ of pulse energy is converted into the third harmonic wave.

This is by itself not a strong drawback for this method, because if the collimating lens, the dichroic mirror, any THG-filters as well as the THG detection camera are properly aligned the signal to noise ration is sufficient enough for the SBTHG method to reach the same measurement accuracy as the confocal backreflection detection method (for an ideal alignment), namely $10 \%$ of the rayleigh length of the focused processing beam. However, in order to be able to align the SBTH based detection setup properly at least some THG signal must be detectable or measurable. The low conversion efficiency makes this task pretty difficult. One possible way to increase the efficiency would be either to increase the laser power or switch to a laser with shorter pulse durations. However, in the first case the surface might get damaged while in the latter case would counter the benefit of using the processing laser for alignment. Besides these less-than-optimal solutions the conversion efficiency might be increased by the selection of a material with higher conversion efficiency at least for the initial stage of the alignment. However, it is not clear which material is best suited to this task. Theoretical models describing SBTHG give the following relationship between the material constants and the conversion efficiency $\eta_{T H G}^{[4]:} \eta_{T H G} \propto \frac{\left(\chi_{S}^{(3)}\right)^{2}}{\varepsilon_{F} \cdot \varepsilon_{T H G}}$

with $\chi_{S}^{(3)}$ the surface susceptibility tensor of $3^{\text {rd }}$ degree, $\varepsilon_{T H G}$ and $\varepsilon_{F}$ the dielectric constant of the material at the wavelength of the third harmonic and the fundamental respectively. However, the practical use of eq. 1 might be fairly limited, as the dielectric constants depend on the refraction index and absorption coefficient of the material, and the surface susceptibility tensor is known only for very few materials and exhibits, even so, generally a very large measurement uncertainty. Experiments analyzing SBTHG point to the fact that the jump of the refractive index at a surface or the resulting electric field gradient has an impact on the THG conversion efficiency [5] or that the absorption coefficient has an impact on the THG conversion efficiciency [12]. However, from these experiments it is not clear if the main factor that determining is material-given by its inner molecular- and crystal-structure $\left(\chi_{s}{ }^{(3)}\right.$ dependent) or if it is rather determined mainly by the macroscopic refractive index and absorption coefficient of the material ( $\varepsilon$-dependent), since this has the largest impact on the electric gradient field at the surface. Moreover, if the THG conversion efficiency depends on the dielectric constant it is not clear what kind of relationship exists between $\varepsilon$ and the conversion efficiency (linear/nonlinear). In order 
to answer some of these questions, the following experiments were carried out.

\section{Impact of materials and their combinations on the strength of SBTHG}

The pulse energy of the fundamental used for the experiments ranged from $0.02 \mu \mathrm{J}$ up to $1 \mu \mathrm{J}$. The repetition rate was adjusted using a pulse picker (without affecting the pulse energy) from $1 \mathrm{kHz}$ to $1 \mathrm{MHz}$ in order to raise the signal strength of the third harmonic above the detection threshold of the THG-camera. This way, the THG conversion efficiency can be calculated without the need to use extremely sensitive power detection equipment. The materials available for this experiments were double-side polished silicon $(\mathrm{Si})$, fused silica $\left(\mathrm{SiO}_{2}\right)$ and sapphire $\left(\mathrm{Al}_{2} \mathrm{O}_{3}\right)$. The refractive indexes for both the fundamental and third harmonic wave as well as the thickness of the samples is given in table 1 . The attentive reader will notice that the fundamental at $1064 \mathrm{~nm}$ is actually outside the transmission window of silicon which normally extends from around $1.2 \mu \mathrm{m}$ to far infrared wavelengths. Even more curious is the fact that we try to observe the third harmonic at $355 \mathrm{~nm}$ in a transmission setup well knowing that any wavelength in this region will experience a very strong absorption in silicon. That SBTHG is possible, albeit on thin silicon samples, is shown in fig. 3 which shows a comparison of the confocal backreflection and the SBTHG based detection methods for fused silica and silicon.

Table 1 Thickness and refractive indexes for fundamental and third harmonic of the analyzed materials.

\begin{tabular}{cccc}
\hline Material & thickness & $1064 \mathrm{~nm}$ & $355 \mathrm{~nm}$ \\
\hline $\mathrm{Si}$ & $0.398 \mathrm{~mm}$ & 3,6 & - \\
$\mathrm{SiO}_{2}$ & $1.058 \mathrm{~mm}$ & 1,450 & 1,476 \\
$\mathrm{Al}_{2} \mathrm{O}_{3}$ & $1.025 \mathrm{~mm}$ & 1,755 & 1,755 \\
\hline
\end{tabular}

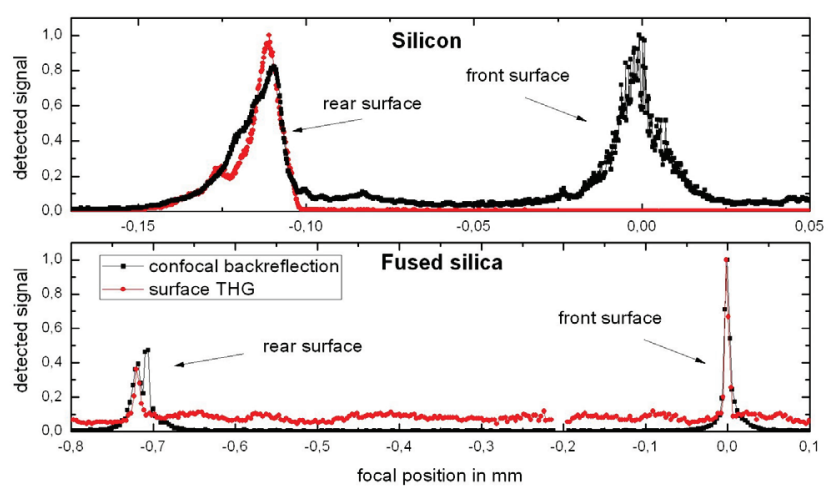

Fig. 3 Comparison between the confocal backreflection and the SBTHG based detection methods for fused silica and silicon.

As can be seen in fig. 3 the confocal backreflection method is able to detect the positions of the front and the rear surface for fused silica as well as silicon. This means that the absorption of the fundamental at $1064 \mathrm{~nm}$ is small enough and the thickness of the silicon sample thin enough that even double crossing of the sample (necessary for the detection of the rear surface in backreflection) is possible without too large losses. However, the difference between the materials becomes obvious for SBTHG in transmission, where for silicon the THG signal can be observed only for the rear surface while in the case of fused silica both surfaces are visible in the transmitted third harmonic signal. Even more curious is the fact that the SBTHG at the rear side of the silicon is strong enough to excite fluorescence on a white paper that is visible to the naked eye while the SBTHG from fused silica (at the same laser settings) is too weak to be observed. As can be seen, the third harmonic signal from the front surface of fused silica is stronger than from its rear. The main reasons for this are spherical aberrations that occur due to deep focusing and lead to intensity dilution of the focal spot [6]. Without doubt the third harmonic signal would have been much stronger for the silicon sample, since at the front surface neither absorption nor spherical aberrations decrease the intensity within the focal spot. Even so, it seems reasonable that the SBTHG from silicon's front surface should be observable in a SBTHG reflection setup. However, such a setup must fulfill more technical constraints than that of SBTHG in transmission because the processing optics must exhibit good transmission and small chromatic aberrations also for the third harmonic wave instead only for the fundamental.

Of course, the three materials in table 1 are too few for representative measurements of the influence of material properties on the SBTHG conversion efficiency. In order to increase the number of material combinations the samples were optically contacted. This means the surfaces of the different materials were brought close enough together that Van-der-Waals forces might act in between resulting in a distance of typically $10 \mathrm{~nm}$ between the surfaces [7]. Transparent dielectrics of the same material bonded in this way do not exhibit any backreflection from the optically contacted region - the material appears to be a single bulk material. Obviously the gap is small enough that the evanescent field that occurs while a reflection takes place at the surface of a transparent dielectric material transforms its potential energy fully by exciting a propagating electromagnetic field in the closely adjacent material. Consequently, the residual evanescent field at such an optically contacted interface should be very close to zero (assuming same materials) and, therefore, the field gradient should be also zero preventing the generation of surface based third harmonic wave. Under these considerations optically contacted samples of different materials seem to be ideally suited to investigate whether the conversion efficiency is mainly $\chi_{s}^{(3)}$ - or $\varepsilon$-dependent.
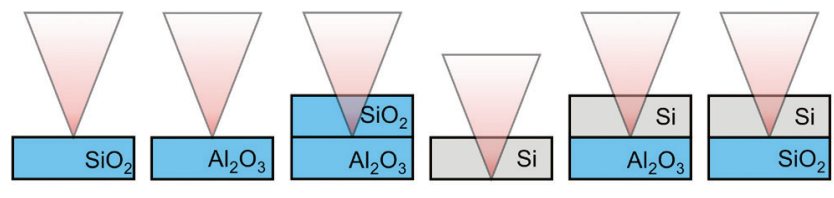

Fig. 4 Arrangement of investigated materials and material combination.

Fig. 4 shows the beam-sample alignment of the investigated materials and materials combinations. In principle, the combination of $\mathrm{SiO}_{2} / \mathrm{Al}_{2} \mathrm{O}_{3}$ allows two measurements, one with $\mathrm{SiO}_{2}$ on top and the other with $\mathrm{Al}_{2} \mathrm{O}_{3}$ on top. However, only the combination with $\mathrm{SiO}_{2}$ on top has been investigated, because the reflection coefficient of the 
$\mathrm{SiO}_{2} / \mathrm{Al}_{2} \mathrm{O}_{3}$ interface is independent on the irradiation side. Furthermore, having $\mathrm{SiO}_{2}$ on top reduces the losses due to reflections at the entrance facet. As focusing lens a microscope objective designed for infrared with a numerical aperture of 0.55 was used resulting in a focal spot diameter of roughly $3 \mu \mathrm{m}$. The efficiency of SBTHG was determined by measuring the pulse energy of the fundamental and detecting the third harmonic by a linear camera sensor. The signal of the THG camera was then normalized to the pulse energy impinging on the interface generating the third harmonic. In order to calculate the pulse energy the Fresnel reflectivity of surfaces traversed by the fundamental and situated in front of the TH-generating interface were calculated by using the refractive indexes shown in table 1 which were derived for fused silica and sapphire from the Sellmeier-equation using coefficients given in $[8,9]$. The refractive index of silicon was calculated by measuring the geometrical sample thickness dividing it by the optical sample thickness measured by the confocal backreflection method. The same was done for the THG camera signal if the TH-generating interface of the third harmonic had to cross another surface before leaving the sample. Additionally, the absorption of the fundamental in silicon was calculated using the Lambert-Beer-law and the absorption coefficient of silicon for $1064 \mathrm{~nm}$ given in [10]. Initial analysis, where the normalized SBTHG signal was plotted against the difference of the refraction coefficients of the THgenerating interface materials has shown some monotonic increase, however, a clear dependency could not be found. If plotted against the Fresnel reflection coefficient of the $\mathrm{TH}$-generating interface (calculated for normal incidence for simplicity reasons), a clear, probably even linear dependence of the SBTHG was found. These results are shown in fig. 5.

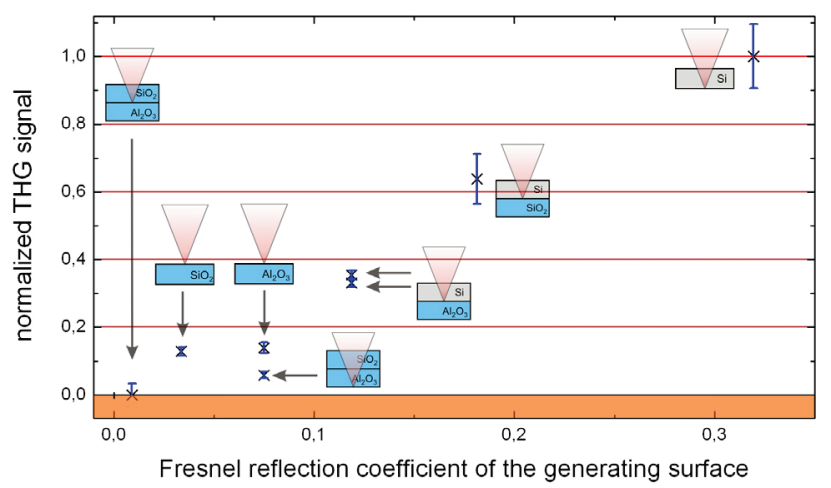

Fig. 5 Measured SBTHG signal plotted against the Fresnel reflection coefficient of the TH-generating surface. The sample-beam alignment is given as inset for each data point.

Fig. 5 shows that the SBTHG seems to depend linearly on the Fresnel reflection coefficient of the TH-generating surface. Some data points seem to be outliers to this tendency such as the interface between fused silica and sapphire or the rear side of the fused silica/sapphire sample. In the first case no distinctive signal could be measured from the interface - the noise of the SBTHG was probably too large. Experiments described in literature show that the third harmonic signal can be measured also for much smaller refractive index differences (or Fresnel reflectivi- ties) [11], however, the pulses used therein had much shorter (femtosecond) pulse durations than in our case. It seems plausible that shorter pulse duration can improve the conversion efficiency so that also surfaces with small Fresnel coefficients can be detected. The outlier at the rear side of sapphire of the fused silica/sapphire sample can be attributed with high probability to spherical aberrations that occur due to the deep focusing of the relatively high NA objective used for these experiments [6].

The obviously linear dependence of the generation efficiency on the reflection coefficient of the TH-generating interface suggests that the SBTHG conversion efficiency is determined rather by the refractive indexes of the involved materials ( $\varepsilon$-dependent) than by some material specific properties $\left(\chi_{s}^{(3)}\right.$-dependent). This can be helpful to determine the suitability of optical materials (without known SBTHG conversion efficiency) for an easy alignment of the SBTHG based surface detection.

The results show that higher reflection coefficients yield a stronger SBTHG signal. This means that doubleside polished optical materials with high refractive index such as sapphire, heavy flint glass, diamond, yttrium aluminum garnet (YAG), yttrium orthovanadate $\left(\mathrm{YVO}_{4}\right)$, lithium niobate [5], titanium dioxide [5] should be used in combination with air. If lasers in the infrared or far infrared region are used, even semiconducting materials can be applied since they posses an even larger refractive index than the aforementioned materials. As shown above, using silicon as an example the semiconductor materials can be even useful for SBTHG at wavelengths that lie outside the actual transmission window of the material as long the samples are thin enough.

\section{Conclusions and outlook}

In this work the efficiency of surfaced based third harmonic generation (SBTHG) was analyzed for different materials and their optically contacted combinations. This can be of importance for an easy alignment of the SBTHG based surface detection method which offers the possibility to detect the absolute focus position of the processing laser beam with respect to the sample's surface with high accuracy and without damaging the sample. Thus this method offers a high applicability as a calibration method for the confocal backreflection surface detection. However, one of the drawbacks of the SBTHG based surface detection is the low conversion efficiency which makes a proper alignment difficult which is nonetheless necessary to achieve a sufficiently high signal to noise ratio of the surface generated third harmonic.

The results presented herein show that the efficiency of the SBTHG depends strongly (and probably in a linear way) on the reflection coefficient of the TH-generating interface. Consequently, in order to achieve a high conversion efficiency (even as a first step in the alignment of the SBTHG setup) a material should be selected which has a rather high refractive index. Even partially absorbing materials can be considered if the sample thickness is small enough.

The findings described in this work may also offer new opportunities for designs on efficient THG. Since the conversion efficiency depends strongly on the reflection coefficient of optically contacted interfaces consisting of different materials also a multiple dielectric layer system may 
appear feasible where the layer materials are transparent to both the fundamental and third harmonic wave, have a large refractive index difference for the fundamental and where their thickness is chosen such that the third harmonic can be generated in a coherent way.

\section{Acknowledgments}

This work was partially supported by Erlangen Graduate School in Advanced Optical Technologies, FriedrichAlexander University Erlangen-Nürnberg.

\section{References}

[1] Cvecek, K.; Miyamoto, I.; Strauss, J.; Bui, V.; Scharfenberg, S.; Frick, T.; Schmidt, M.: Strengh of Joining Seams in Glass Welded by Ultra-fast Lasers Depending on Focus Height. JLMN-Journal of Laser Micro/Nanoengineering, Vol. 7, No. 1, 2012.

[2] Quentin, U.; Leitz, K.-H. Alexeev, I.; Schmidt, M.: Application of Gaussian Optical Tweezers for Ultrafast Laser Assisted Direct-write Nanostructuring, JLMN-Journal of Laser Micro/Nanoengineering, Vol. 7, No. 2, 2012.

[3] Alexeev, I.; Strauss, J.; Gröschl, A.; Cvecek, K.; Schmidt, M.: " Laser focus positioning method with submicrometer accuracy," Appl. Opt. 52, No.3, 2013

[4] G. Berkovic, R. Superfine, P. Guyot-Sionnest, Y. R. Shen, and P. N. Prasad, "Study of diacetylene monomer and polymer monolayers using second- and thirdharmonic generation", J. Opt. Soc. Am. B 5, pp. 668-673 (1988).

[5] Tsang, T.: "Optical third-harmonic generation at interfaces”, Phys. Rev. A, Vol. 52 No. 5, (1995).

[6] K. Cvecek, I. Miyamoto, M. Adam, M. Schmidt: Effects of spherical aberrations on micro welding of glass using ultra short laser pulses, Proc. LANE 2012, Fuerth, Germany, Physics Procedia, Volume 39, (2012), pp. 563-568

[7] V. Greco, F. Marchesini, and G. Molesini, "Opticalcontact and van der Waals interactions: the role of the surface topography in determining the bonding strength of thick glass plates", J. Opt. A: Pure Appl. Opt. 3, 85-88 (2001).

[8] I. H. Malitson, "Refraction and Dispersion of Synthetic Sapphire", J. Opt. Soc. Am., Vol. 52, No. 12, (1962), pp. $1377-1379$

[9] SCHOTT Technical Information, "Refractive index and dispersion", TIE29, Download: www.schott.com/advanced_optics/english/download/ti e-29_refractive_index_v3.pdf

[10] G. E. Jellison, Jr., D. $\bar{H}$. Lowndes, "Optical absorption coefficient of silicon at $1.152 \mu$ at elevated temperatures", Appl. Phys. Lett., Vol. 41, No. 7, 1982, pp. 594-596.

[11] C. B. Schaffer, A. Brodeur, J. F. García, and E. Mazur, "Micromachining bulk glass by use of femtosecond laser pulses with nanojoule energy," Opt. Lett. 26, 9395 (2001).

[12] G. Veres, S. Matsumoto, Y. Nabekawa and K. Midorikawa, "Enhancement of third-harmonic generation in absorbing media", Appl. Phys. Lett. 81, 2002, pp. $3714-3716$ 